\title{
ACTIVIDAD FÍSICA Y DISCAPACIDAD: UN ESTUDIO CUALITATIVO CON MUJERES EN UN GIMNASIO ADAPTADO
}

\author{
ATIVIDADE FÍSICA E INCAPACIDADE: UM ESTUDO QUALITATIVO COM \\ MULHERES EM UM GINÁSIO ADAPTADO
}
PHYSICAL ACTIVITY AND DISABILITY: A QUALITATIVE STUDY ON WOMEN AT AN ADAPTED GYM

Víctor Pérez-Samaniego*, Elena López-Cañada*, Javier Monforte*

\begin{abstract}
Palabras clave: Bienestar social. Autonomía personal. Facilitadores. Barreras.

Resumen: Este estudio explora cómo un grupo de mujeres con discapacidad $(n=6)$ experimentan y perciben la práctica de actividad física en un gimnasio adaptado. Se utilizaron entrevistas semiestructuradas como instrumento de recogida de datos cualitativos y se realizó un análisis temático inductivo. Los resultados se agruparon en torno a cuatro categorías temáticas emergentes: condición física y autonomía personal; papel paliativo del ejercicio; bienestar psicológico; relación social y apoyo. Destaca la experiencia satisfactoria de las participantes, en la que predomina una valoración positiva de procesos autorregulativos, la concepción social-relacional de la actividad física y la relevancia del gimnasio adaptado como facilitador ambiental y social.
\end{abstract}

Palavras chave: Bem-estar social. Autonomia pessoal. Barreiras. Facilitadores.

Keywords:

Social Welfare.

Personal autonomy. Facilitators. Barriers.

Resumo: Este estudo explora como um grupo de mulheres com incapacidade $(n=6)$ experimenta e percebe a prática da atividade física em um ginásio adaptado. Foram realizadas entrevistas semiestruturadas como instrumento de coleta de dados qualitativos e uma análise temática indutiva. Os resultados foram agrupados em torno de quatro emergentes categorias temáticas: aptidão e autonomia pessoal; papel paliativo de exercício; bem-estar psicológico; relação social e apoio. Ele destaca a experiência bem sucedida dos participantes, que é dominada por uma avaliação positiva dos processos de autorregulação, concepção social-relacional da atividade física e a relevância do ginásio adaptado como facilitador ambiental e social.

Abstract: This study explores how a group of disabled women $(n=6)$ experience and perceive physical activity practice in an adapted gym. Semi-structured interviews were used to collect qualitative data, and a thematic inductive analysis was carried out. Results were grouped under four emergent thematic categories: physical fitness and personal autonomy; the palliative role of exercise; psychological wellbeing; social relation and support. Participants' satisfaction with the experience is highlighted, predominating positive appraisal of self-regulation processes, social-relational conception of physical activity, as well as the relevance of the adapted gym as an environmental and social facilitator.
*Universidad de Valencia. Valencia, España.

E-mail: victor.m.perez@uv.es; Elena.lopez-canada@uv.es; javier.monforte@uv.es

Recebido em: 22-10-2016 Aprovado em: 03-03-2017

(c) (i) () Licence 


\section{INTRODUCCIÓN}

En España, viven aproximadamente 3,85 millones de personas con discapacidad (PCD) (8,5\% de la población), el 60\% de las cuales son mujeres (INSTITUTO..., 2008). Las evidencias sobre los peligros de la inactividad física y los beneficios que la práctica regular de ejercicio físico puede aportar a las PCD son fuertes y abundan en la literatura científica (HAMMELL, 2007; MARTIN GININS, 2010; RIMMER; SCHILLER; CHEN, 2012; STEPHENS; NEIL; SMITH, 2012). Sin embargo, las PcD son más sedentarias que el resto de la población. Esto se debe, en parte, a la presencia de barreras personales, sociales y ambientales que dificultan el desarrollo de un estilo de vida activo (MARTIN GINIS et al. 2016).

Este estudio aborda las experiencias de actividad física en tiempo de ocio (AFTO) practicadas por un grupo de mujeres con discapacidad en un gimnasio adaptado. Si bien se acepta que el género es un aspecto relevante a la hora de comprender los significados de la práctica de AFTO, existen pocos y son relativamente recientes los trabajos cualitativos que centren su foco de atención en mujeres con discapacidad (EVANS; ALLEN-COLLINSON; 2016). Por otra parte, los espacios resultan determinantes para la participación, puesto que la ausencia de zonas accesibles para la práctica de AFTO supone una barrera sustancial, directamente asociada a otras barreras como la falta de asistencia profesional, el equipamiento inapropiado y las actitudes negativas de los otros (RICHARDSON; SMITH; PAPATHOMAS, 2016). En este sentido, los gimnasios adaptados son entornos potencialmente adecuados para que las $\mathrm{PcD}$ practiquen AFTO, ya que son concebidos y diseñados con tal fin, por lo que no constituyen una barrera en sí misma (RICHARDSON; SMITH; PAPATHOMAS, 2016). Con todo, su influencia en las experiencias de las PcD todavía está por determinar.

Enmarcada en el interés por identificar y comprender mejor los aspectos clave que facilitan o dificultan la práctica de AFTO de las PcD, la pregunta a la que se busca dar respuesta con este trabajo es la siguiente: ¿cómo experimentan y perciben la AFTO un grupo de mujeres con discapacidad usuarias de un gimnasio adaptado?

\section{MÉTODO}

\subsection{Procedimiento y participantes}

El estudio se llevó a cabo en un gimnasio diseñado y gestionado por una asociación de $\mathrm{PcD}$ al que acuden personas de todas las edades con diferentes tipos de discapacidad motora, psicomotora o condición crónica. La gran mayoría de sus usuarios se desplaza en silla de ruedas. Las instalaciones son perfectamente accesibles y los equipamientos están diseñados o adaptados para su uso por PcD. Todo el personal, incluidos los monitores deportivos, tienen una formación específica sobre ejercicio adaptado. Su función es asistir a las personas usuarias en sus necesidades y orientarles acerca de la práctica de ejercicio, que habitualmente realizan de manera autónoma.

ELC (siglas de coautora) trabajó como voluntaria durante dos años en dicho gimnasio, cumpliendo funciones de monitora durante unas diez horas semanales. A raíz de dicha colaboración surgió el interés por comprender mejor las experiencias de las mujeres 
con discapacidad que practican ejercicio físico con regularidad. Tras una primera toma de contacto, se elaboró un proyecto de investigación que obtuvo el consentimiento de la dirección del gimnasio y la aprobación del comité ético de la Universidad X. La solicitud de colaboración a las participantes se realizó tras un año de permanencia de ELC en el gimnasio, siguiendo la estrategia que Schwandt (1997) denomina muestreo en el campo. Durante este tiempo, la investigadora tuvo la oportunidad de colaborar, conocer y ganar progresivamente la confianza de la gran mayoría de usuarias, llevando durante su permanencia un diario en el que reflejaba aspectos que consideraba potencialmente interesantes para la investigación, incluyendo la accesibilidad, disponibilidad y voluntad de compartir experiencias de potenciales participantes (SPARKES; SMITH, 2014).

Participaron finalmente seis mujeres de entre 30 y 47 años $(M=36.5 ; D E=5.96)$ con diferentes tipos de discapacidad. Dos de las participantes tenían ataxia de Friedrich (AF), otras dos esclerosis múltiple (EM), una de tipo recidivante-remitente y otra progresiva primaria. Las dos restantes presentaban paraparesia espástica progresiva (PEP) y lesión plexo-braquial (LPB) respectivamente. En todos los casos, la discapacidad fue adquirida (hace entre 7 y 26 años). Las mujeres acudían al gimnasio un mínimo de dos días a la semana y un máximo de cinco, una media de dos horas por sesión. Excepto la participante con LPB, el resto precisaba de silla de ruedas para desplazarse.

Los datos del estudio se obtuvieron mediante entrevistas semiestructuradas. Inicialmente, las entrevistas partían de un guion elaborado a partir de temas recogidos en una metasíntesis sobre la calidad de vida en PcD (HAMMELL, 2007), así como de la propia experiencia de ELC como colaboradora en el gimnasio. Algunos ejemplos de temas y preguntas serían: la discapacidad (ej.: ¿Podrías contarme cómo es tu día a día? ¿En qué aspectos incide más tu discapacidad?); percepciones y valoraciones sobre la AFTO (ej.: ¿Qué te aporta hacer ejercicio? ¿Qué es lo que más te gusta de hacerlo? ¿Y lo que menos? ¿Sientes que tienes control sobre tu práctica?); relaciones (ej.: ¿Cómo es tu relación con el resto usuarios? ¿Y con los monitores?); buenos y malos días (ej.: En relación con el ejercicio ¿podrías contarme cómo es para ti un buen día? ¿Y un mal día?). La formulación de las preguntas fue flexible, variando en cada entrevista en función de aspectos introducidos por las propias participantes.

Todas las mujeres a las que se les propuso participar accedieron a conceder una entrevista. Su duración fue de aproximadamente una hora y media, y se realizaron de forma individual, en un despacho separado de la zona de práctica deportiva. Previamente, las participantes fueron informadas del propósito de la investigación, de las medidas de confidencialidad y anonimato (todos los nombres propios utilizados en este estudio son pseudónimos), así como de la posibilidad de cesar en su participación cuando lo consideraran oportuno, sin dar más explicación. Otras preocupaciones éticas tuvieron que ver con posibles dilemas y consecuencias que podían derivarse del encuentro entre personas con y sin discapacidad con propósitos de investigación (MORRISEY, 2012). En particular, ELC, mujer joven que no sufre ninguna discapacidad, procuró en todo momento mostrarse respetuosa con la realidad concreta de cada mujer, evitando valoraciones, consejos, juicios de valor 0 expresiones que pudieran interpretarse como intrusivas. Al tiempo, intentó mostrarse cercana y empática, especialmente cuando se tocaban o emergían temas sensibles. Ante cualquier reacción o circunstancia delicada, la entrevistadora aprovechó su condición de monitora para dar su apoyo personal y hacer un seguimiento de los temas más allá de la entrevista. Este 
tipo de cuestiones no han sido incluidas en este estudio, que se limita a los datos obtenidos en las entrevistas, tal y como se convino con las participantes. Todas las entrevistas fueron grabadas y su transcripción literal fue enviada a cada participante para que diera su aprobación o realizara las matizaciones o correcciones oportunas a su contenido.

\subsection{Análisis}

Los datos obtenidos se analizaron temáticamente de forma inductiva. El análisis temático inductivo se centra en buscar similitudes y diferencias en el conjunto de los datos, con el fin de identificar de manera flexible y directa patrones interpretativos (BRAUN; CLARKE, 2006). Esta forma de análisis resulta especialmente adecuada para captar impresiones heterogéneas sobre una misma realidad -como es el caso de mujeres con distintos tipos y experiencias con la discapacidad y la práctica de ejercicio-, y abrir su discusión a diversos marcos interpretativos. La flexibilidad del análisis temático facilita asimismo identificar casos excepcionales 0 datos singulares (casos negativos) que contrastan con los patrones interpretativos y que, por tanto, ayudan a comprenderlos, ponderarlos y darles sentido.

Los investigadores, de forma reiterada y sistemática, leyeron, reorganizaron e hicieron comentarios descriptivos y exploratorios sobre los datos, subrayando las frases e ideas recurrentes y los conceptos clave. En esta fase preliminar, el análisis se centró en identificar los temas que aparecían en los datos y en realizar tentativas de conexión con la literatura. Finalmente, los resultados se agruparon en torno a cuatro categorías temáticas: condición física y autonomía personal; papel paliativo del ejercicio; bienestar psicológico; relación social y apoyo.

\section{RESULTADOS Y DISCUSIÓN}

\subsection{Condición física y autonomía personal}

Existe evidencia de que la mejora o el mantenimiento de la condición física mejora el estado físico general, reduce el riesgo de lesiones e incide en la prevención de afecciones secundarias en PcD (HICKS et al. 2011). La mejora en la "forma física" (expresión utilizada por las participantes) y sus efectos beneficiosos fue un tema mencionado en todas las entrevistas, valorando siempre positivamente los efectos del ejercicio en su estado físico.

De entre las numerosas y diversas valoraciones positivas de la AFTO en su condición física, las participantes, en su mayoría usuarias de sillas de ruedas, destacan muy especialmente la importancia que para ellas tiene la mejora de la fuerza, sobre todo la de los brazos:

Realizo ejercicio para la masa muscular, para no ir perdiendo... porque yo las piernas pues eso, no tengo movilidad. Entonces yo los brazos, que los tengo bien, pues eso es lo que tengo yo ahora que fortalecer, para que la masa muscular no se me vaya y los tenga fuertes, porque claro para mí, mis piernas y mis pies... son mis brazos, que es con lo que lo hago todo. Para mí es muy importante. (Pepa, EM)

[Con el ejercicio] me encuentro mejor, tengo un poco más de fuerza, que yo antes no me podía levantar de la cama yo sola y ahora sí que me puedo levantar y 
porque como pesa... es que son dos puertas súper gordas y pesan un huevo [sic.]. (Marisol, PEP)

En las citas se aprecia que para las participantes tener "brazos fuertes" implica, sobre todo, ganar movilidad. Es decir, el valor concedido a la fuerza se asocia fundamentalmente con la búsqueda de autonomía en su vida cotidiana. Esta manera concreta de concebir la importancia de la fuerza concuerda con recientes trabajos en los que se destaca la búsqueda y disfrute de la autonomía personal como uno de los principales beneficios del ejercicio físico, así como un potente facilitador para su práctica (NEWITT; BARNETT; CROWE, 2016). En el caso concreto de las mujeres, Hallberg et al. (2010) incluyen la práctica de ejercicio entre lo que denominan estrategias optimistas para el mantenimiento de la autonomía e independencia. Las mujeres que utilizan este tipo de estrategias para hacer frente a la discapacidad tienden a considerar manejable su condición crónica y, desde la aceptación de sus limitaciones, piensan que algo pueden hacer para ganar control sobre sus vidas.

La noción de empoderamiento sirve para enmarcar el sentido y la función de esta estrecha relación entre condición física y autonomía personal. Como señalan Devís y Pérez-Samaniego (2001), empoderar significa, literalmente, ganar poder para, a pesar de las dificultades, intentar vivir la vida tal y como se desea. El significado específico que las participantes otorgan a la fuerza refleja cómo se producen e interactúan la mejora de las aptitudes y los procesos propios del empoderamiento personal. Para las participantes, ganar fuerza es ganar autonomía. Y, como se verá más adelante, todo lo que tienen que hacer para lograrlo, propicia la activación de procesos que, en última instancia, son una manifestación de la propia autonomía personal.

\subsection{Papel paliativo del ejercicio: Reducción del dolor y ralentización del deterioro físico}

El dolor suele acompañar a las PcD (SPARKES; SMITH, 2008). Muchas mujeres con lesión medular dicen sentir un dolor constante, sobre todo en la espalda, que describen como terrible u horrible (HALLBERG et al. 2010). Además, en muchos casos el temor a la aparición de episodios de dolor agudo conlleva ansiedad y acaba dominando sus vidas. Precisamente la disminución o el alivio del dolor sería uno de los aspectos al que más se refieren las participantes:

Luego tú misma te das cuenta que cuando haces ejercicio estás mejor, a nivel de lo que te comentaba, de dolores de espalda y eso, te sientes mejor, te sientes más fuerte [...] Pero, es verdad, [mi cuerpo] es muy agradecido y sí que a nivel de la espalda, sobretodo tengo mejoría. Tengo dolor, pero mucho menos. (Lorena, AF)

El ejercicio creo que sí que me lo alivia [el dolor], porque yo vengo aquí y... Es que lo que sea ya me noto...Yo salgo de aquí y me noto súper recta. [...] Y sigo teniendo dolor, pero quizás si dejara de hacer ejercicio o no llevara esta vida tan activa me dolería más... (Marta, LPB)

El dolor es una sensación incapacitante que limita o retrae a las $\mathrm{PcD}$ de la práctica de ejercicio. Tener en cuenta la disminución progresiva del dolor y del temor al dolor puede jugar un papel muy positivo en la promoción de la actividad física, ya que puede motivar a las PCD a mantenerse activas, obteniendo así mayores beneficios saludables. De forma similar, puede hacer entender a los monitores y cuidadores el fuerte impacto del dolor como condicionante inicial de la práctica de AFTO en PCD, así como el valor clave que juega su progresiva disminución como motivador (SPARKES; SMITH, 2008). 
Otro de los efectos paliativos asociados a la práctica regular de ejercicio a los que se refieren las participantes es la detención, disminución o ralentización del deterioro físico (STEPHENS; NEIL; SMITH, 2012). Dos de ellas ponen especial énfasis en este tipo de argumentos para justificar su necesidad de hacer ejercicio:

El ejercicio físico del gimnasio me gusta en el sentido de que no me queda otra si quiero mantenerme bien, no tengo más remedio y ya está [...] intento mantenerme lo más posible para poder mantener ese modo de vida que a mí me gusta. (Lorena, AF)

Hay que hacer deporte, claro, aunque no me guste, para no perder cada vez más. Y más mi enfermedad que es degenerativa. [...] [Con el ejercicio] pierdo menos, pierdo más despacio. Yo sé que mejor no voy a estar, pero pierdo menos. Antes, pues eso, en seis años a lo mejor he perdido por poner en una escala, he perdido 50 en cinco años y en tres o dos he perdido, en vez de 10 por año, he perdido 5. Sí, he perdido más despacio. (Esperanza, EM)

Estas citas, con sus reiteradas referencias a "la enfermedad" y la necesidad de ejercitarse para mitigar efectos adversos, evocan una concepción del ejercicio propia del modelo médico de discapacidad. Como señala Thomas (2007), el modelo médico contribuye a configurar los significados de la discapacidad y ejercicio físico en torno a sus consecuencias biológicas experimentadas a nivel individual. El ejercicio físico pasa a ser concebido un medicamento que debe ser prescrito con arreglo a problemas de salud propios de PcD. La función del practicante se limitaría a realizar la actividad prescrita a fin de preservar su físico, mitigar el dolor o prevenir el deterioro funcional.

Para las $\mathrm{PcD}$, esta concepción de ejercicio como medicina puede resultar deseable a corto plazo, de cara a ser conscientes del papel beneficioso del ejercicio en la prevención de condiciones secundarias asociadas a la inactividad física. No obstante, como recuerdan Smith y Perrier (2014), también resulta problemática, ya que al situar el "problema" y la "solución" de la discapacidad en el individuo se corre el riesgo de medicalizarla, responsabilizando (e indirectamente culpabilizando) a las PcD de su inactividad física. Tiende así a olvidarse 0 relegarse la importancia de una respuesta social necesaria para que las $\mathrm{PcD}$ puedan realizar ejercicio físico de forma regular y, por tanto, beneficiarse de sus efectos paliativos.

\subsection{Bienestar psicológico}

En la población adulta en general la práctica regular de ejercicio físico ayuda a relajarse, despejar la mente, evitar el estrés, mejorar la autoestima y mantener una actitud positiva ante la vida (STATHI; FOX; MCKENNA, 2002). En numerosas ocasiones, las mujeres entrevistadas aluden a beneficios psicológicos similares al referir cómo la AFTO les genera sensaciones y emociones positivas con expresiones como éstas: "el ejercicio me aporta bienestar e incluso estoy con más ánimo" (Elisa, AF); "el ejercicio me relaja, estoy mucho mejor. [...] Me siento más alegre y mejor conmigo misma" (Marisol, PEP). Resultados similares se recogen en trabajos en los que se destaca que el ejercicio regular contribuye a aumentar la autoestima y la autoconfianza de las $\mathrm{PcD}$, así como a disminuir la incidencia de trastornos de depresión y ansiedad (MARTIN GINIS et al. 2016).

Además de los beneficios psicológicos ligados al ejercicio en sí, Hammell (2007) subraya la importancia de cambiar de entorno, así como de tener ocupaciones y rutinas como aspectos clave para el bienestar psicológico de las PcD. Estas cuestiones se ven reflejadas en las siguientes citas: 
Ahora que vengo al gimnasio, estoy más tiempo fuera de casa y eso se nota. En el sentido de que, por ejemplo, mi caso...si yo estoy encerrada mucho tiempo en casa, es cuando empiezo a comerme la cabeza por una simple tontería. [...] Y para llegar al gimnasio tengo que coger dos autobuses, y ahí sí que me siento satisfecha. [...] Vengo de lunes a viernes de cuatro a ocho [de la tarde], que estaré dos horas y media o así haciendo [ejercicio] [...] y cuando entro, sé lo que tengo qué hacer y lo hago. (Elisa, $\mathrm{AF}$ )

Yo vengo en el autobús especial, porque me recogen y me traen todas las veces que venimos. [...] Vengo dos días, lunes y miércoles, desde las cuatro y media que llegamos, hasta las siete y media. [...] Yo siempre cuando vengo, empiezo a hacer bicicleta, siempre hago una hora y después triciclo... voy yo sola, a mí sólo me ayudan para subir a la bicicleta y ya está. Luego voy yo por ahí sola. También hago gomas [un ejercicio adaptado de flexo-extensión de brazos], y a veces en la colchoneta, para hacer equilibrio... [...] A mí me gusta mucho venir aquí al gimnasio. Así salgo y mejor, me distraigo... porque así no estoy en mi casa todo el día, que sería un agobio total... [...] Muchísimo mejor viniendo al gimnasio, cuanto más salga, mejor. (Marisol, PEP)

La valoración positiva que hacen las participantes de las rutinas y obligaciones autoimpuestas que entraña la AFTO puede ser analizada desde la óptica de la Teoría de la Autodeterminación (RYAN; DECl, 2000). La autodeterminación tiene que ver con el grado en que las personas hacen algo de manera voluntaria. A mayor autodeterminación, las conductas tienden a un mayor grado de autorregulación que, a su vez, suscita sensaciones de competencia y autorrealización. Williams, Smith y Papathomas (2014) destacan que los procesos autorregulados contribuyen especialmente a mejorar la autoestima y la autoconfianza de las PcD. En este sentido, la autorregulación a la hora de hacer AFTO constituye al mismo tiempo un indicador y un potenciador del bienestar psicológico.

Si bien a lo largo de las entrevistas predominan mayoritariamente las valoraciones positivas sobre los efectos psicológicos, dos participantes se refieren puntualmente a su falta de interés y satisfacción por la AFTO:

Yo pienso que no [me gusta hacer ejercicio]. Es una obligación pura y dura. Tengo que venir y vengo. Antes sí que era muy activa, necesitaba tener la cabeza ocupada, pero ahora el ejercicio físico no me ocupa la cabeza. Antes sí... pero esto [el tipo de ejercicio que realiza en la actualidad] es tan básico que no... me ocupa muy poco. Sí, tengo que mover estos músculos y tal... pero es muy poco. (Esperanza, EM)

Hay mucha gente que dice "yo sí que cuando suelto ahí todo, estoy mejor". Yo creo que tengo tan metido que tengo que hacerlo por obligación que no me da tiempo a plantearme nada (sonríe), es verdad. [...] Sí, mucha gente lo dice. Yo no, la verdad. Lo hago [los ejercicios] y eso... lo tengo asumido. (Lorena, AF)

En estos testimonios las participantes aluden a la AFTO como una necesidad autoimpuesta, si bien su realización no parece satisfacerles particularmente. Interpretadas desde la óptica de la Teoría de la Autodeterminación (RYAN; DECl, 2000) estas citas evocan una regulación introyectada, que se asocia a aspectos como la auto-aprobación y la evitación de los sentimientos de culpa. Con respecto a la práctica física, este tipo de regulación es propia de personas que muestran opiniones como "debo hacer ejercicio porque me lo recomienda mi médico" o "me siento mal si no he practicado" (HAGGER; ARMITAGE, 2004). En estos casos, no podemos hablar de una influencia negativa del ejercicio en el bienestar psicológico, pero 
tampoco de una influencia positiva más allá de la compensación de los efectos negativos que tendría no hacerla. Así pues, regulación introyectada puede motivar a realizar AFTO, si bien a expensas de obviar o desdeñar la posibilidad de disfrute y satisfacción personal con su práctica.

\subsection{Interacción personal y apoyo social}

La importancia de las interacciones personales y el apoyo social son aspectos destacados en la literatura sobre discapacidad y actividad física (HAMMELL, 2007; WILLIAMS; SMITH; PAPATHOMAS, 2014). Un aspecto que se destaca positivamente en todas las entrevistas es la relación con los monitores y voluntarios del gimnasio. La siguiente cita ilustra cómo las participantes sienten atendidas sus necesidades, percibiendo además un trato respetuoso y cordial:

En un gimnasio normal no hay nadie disponible para ayudarte a cambiarte, bajarte los reposapiés, colocarte las cintas [para pedalear en bicicleta manual] o llevarte al baño. Cosas así pequeñitas son imposibles [en otros gimnasios]. Y entonces este gimnasio... aquí se cumple [...]. A ver, aparte de que están pendientes, me conocen y saben que me gustan mucho las bromas y bromean mucho conmigo. (Elisa, AF)

Además del trato con monitores y voluntarios, las mujeres entrevistadas subrayan que acudir al gimnasio les permite conocer a otra gente con discapacidad, establecer amistades, compartir puntos de vista y ayudarse entre sí. La edad, el tipo de discapacidad o la clase de problemas compartidos son aspectos destacados a la hora de establecer dicha relaciones entre iguales:

La verdad que muy bueno [el ambiente en el gimnasio] y muy... te sientes como en un ambiente familiar. [...] Además, la mayoría de gente es de mi edad, no sé, cada uno tiene un tipo de discapacidad... nos lo contamos todo. Nos hacemos nuestros puntos de vista... y por ejemplo, Lorenzo [compañero del gimnasio con tetraplejia] me puede decir "pues esto a mí... pues a mí con la pelota, utilizándola, me va bien para esto. Pruébalo y a ver cómo te va a ti. Nos ayudamos" (Elisa, AF).

Yo creo que también es muy importante porque le cuentas tus problemas a la gente por ahí [fuera del gimnasio] y la gente te mira un poco así...claro, es que la gente no te entiende. Pero hablas aquí [en el gimnasio] con la gente y te entienden perfectamente. El otro día un chico me decía de unos problemillas que tenía... luego resulta que tenía una incontinencia terrible, pero claro, con el resto de gente no lo puedes hablar. Y aquí yo le contaba mi experiencia con el pañal y bueno, pues lo hablas con más... no sé, le quitas leña al fuego. Creo que también es importante la relación entre nosotros, por eso, porque hablas de cosas que entiendes, o sea, cosas que a la gente también le pasan, son tus problemas... (Esperanza, EM)

Como puede apreciarse, compartir experiencias con iguales ayuda a obtener información y a motivarse para practicar ejercicio. En ese mismo sentido, Letts et al. (2011) señalan que participar en programas con iguales proporciona sentido a las metas que pueden alcanzarse teniendo lesión medular, así como un estímulo para conseguirlo. Por lo demás, la relación con iguales permite además recabar y proporcionar información incidental sobre otros temas que "con el resto no puedes hablar". El contacto entre personas que llevan distinto tiempo conviviendo con la discapacidad puede contribuir positivamente tanto a los procesos de ajuste de personas que acaban de adquirirla, como al sentido de autoeficacia de personas con más experiencia (DIBB et al. 2014). 
Además de información y estímulo, los iguales proporcionan sostén emocional y buena compañía (PERRIER; SMITH; LATIMER-CHEUNG, 2015). En ese sentido, muchas de las participantes destacan que el gimnasio se convierte en un marco favorecedor de relaciones sociales y de amistad:

A nivel social vienes aquí y te relacionas con más gente que tiene los mismos problemas que tú. Pienso que eso es muy importante, que ya no es por el ejercicio sino por el ambiente, que sí que te aporta... vamos, que no te sientes un bicho raro. Hablas aquí con la gente y te entienden perfectamente. [...] La energía del grupo siempre es mucho más importante que la energía individual. (Esperanza, EM)

En este gimnasio todo se ve más normal, nadie se mira y nadie dice "iHala, mira lo que estás haciendo!", no, pues cada uno hace lo que tiene que hacer y ya está, y eso está guay (sonríe). (Marta, LPB)

Probablemente, sentir que "nadie te mira" y que no se es "un bicho raro" resulta consecuencia de que los usuarios del gimnasio sean mayoritariamente personas con discapacidad. La relación social se ve así favorecida por el hecho de "pasar desapercibidas" y "sentirse comprendidas", lo que hace que nadie se sienta observado ni juzgado por sus deficiencias corporales. La importancia de la corporeidad percibida en relación con los otros pone en evidencia que la discapacidad no solo es un determinado estado físico o impedimento funcional, sino también es una experiencia vivida entre personas, tal y como se destaca desde el modelo social-relacional de discapacidad (THOMAS, 2007). Van de Ven et al. (2008) muestran que las PcD resultan particularmente sensibles a la percepción del rechazo social y las actitudes negativas del público en general lo que, entre otras consecuencias, afecta negativamente a la participación en AFTOs. De ahí que, tal y como plantean Smith y Perrier (2014: 101), lo que la literatura refiere tradicionalmente como eliminación de barreras debiera alcanzar también a "la mirada de los extraños en el gimnasio o [...] a ciertas imágenes o expresiones denigrantes 0 insultantes".

\section{CONCLUSIONES Y CONSIDERACIONES FINALES}

Los resultados de este estudio recogen evidencias y ofrecen interpretaciones acerca de cómo un grupo de mujeres con diversas discapacidades experimentan la AFTO en un gimnasio adaptado. El predominio de impresiones y valoraciones positivas lleva a concluir que, en general, la práctica de ejercicio físico en este entorno resulta para ellas una experiencia satisfactoria. A la hora de caracterizar y comprender cómo se produce y qué implicaciones tiene dicha experiencia satisfactoria en el bienestar de las $\mathrm{PcD}$, los resultados concuerdan con trabajos cualitativos previos. La mejora de la condición física (en concreto de la fuerza), la reducción del dolor y el retraso en el deterioro físico se revelan los principales beneficios del ejercicio a nivel físico (HALLBERG et al. 2010; HICKS et al. 2011; STEPHENS; NEIL; SMITH, 2012). En cuanto al bienestar psicológico, el estudio confirma las valoraciones positivas en aspectos como la relajación y la mejora de la autoestima hechas en otros estudios con PCD (RICHARDSON; SMITH; PAPATHOMAS, 2016). A nivel social y relacional la adecuada atención y cuidado brindado por el personal del gimnasio, así como la información y apoyo que genera la relación entre iguales son aspectos resaltados positivamente (LETTS et al. 2011; DIBB et al. 2014).

Profundizando en los significados e implicaciones de la AFTO para las mujeres, los resultados de este estudio muestran cómo las participantes sitúan en un primer plano aspectos 
procesuales vinculados a sensaciones y vivencias que se producen durante la práctica física, o que se asocian indirectamente con ella (PÉREZ-SAMANIEGO; DEVÍS, 2004). De ahí la importancia que confieren, por ejemplo, a la distracción, la obligación, el cambio de entorno y las rutinas autoimpuestas, así como al hecho de no sentirse juzgadas por la mirada de los otros en el gimnasio adaptado, aspectos todos ellos relativos a la autorregulación y la vivencia de AFTO desde una concepción social-relacional de la discapacidad. Como plantean Williams, Smith y Papathomas (2014), los profesionales de la salud deben identificar mensajes creíbles y efectivos para transmitir los beneficios de la práctica física entre las $\mathrm{PcD}$. Por tanto, estas consideraciones pueden ser útiles para ampliar la comprensión de los beneficios que esperan obtener las mujeres con discapacidad, así como para establecer metas realistas y entender mejor la diversidad de factores a tener en cuenta en la promoción de la AFTO.

También resulta fundamental destacar el papel que juega el gimnasio adaptado como facilitador ambiental y social de la experiencia satisfactoria de las participantes. Cabe subrayar sus cualidades en tanto entorno compuesto por espacios, ritmos, actividades, equipamientos y personas significantes. La interacción entre agentes humanos y no humanos que se da en este gimnasio media positivamente en las vivencias y percepciones que las practicantes tienen de la AFTO, y opera activamente en el desarrollo de aptitudes y procesos empoderadores orientados hacia el desarrollo de la autonomía personal. Estos resultados sitúan los entornos adecuados de práctica como un elemento clave la promoción de la AFTO en PCD. En este sentido, un entorno adecuado no es solo un entorno adaptado sino, además, un ambiente en el que las PcD con discapacidad se sientan capaces y bienvenidas.

Para ello, más allá de la viabilidad o conveniencia de gimnasios exclusiva 0 preferentemente dirigidos a $\mathrm{PCD}$, resulta preciso que los contextos tradicionales de AFTO se hagan más inclusivos. Además de la necesaria eliminación de barreras arquitectónicas, los entornos adecuados deberían contar con equipamientos que todas las personas, incluidas las $\mathrm{PcD}$, pudieran utilizar de forma autónoma o con un mínimo de asistencia. En el caso de actividades organizadas, también sería deseable contar con programas 0 franjas horarias orientadas preferiblemente a $\mathrm{PCD}$, en las que se dispusiera de más personal para atender personas con grandes discapacidades. El personal que trabaja en instalaciones deportivas debería conocer y ser sensible a las necesidades, intereses, posibilidades y gustos de las $\mathrm{PcD}$. Además del conocimiento técnico sobre deporte o ejercicio adaptado, su formación y su preocupación profesional deberían incidir en aspectos referidos al trato adecuado hacia un colectivo especialmente vulnerable.

Las conclusiones e implicaciones de este estudio deben verse con cautela debido las limitaciones que presenta. El hecho de que las entrevistas fueran realizadas por una persona que trabajaba en el gimnasio supuso una gran ventaja a la hora de facilitar el acceso a las participantes y el rapport en las entrevistas, pero pudo también haber introducido un sesgo en la valoración positiva de las experiencias, circunstancia que se intentó evitar preguntando específicamente sobre los aspectos negativos de su experiencia, pese a lo que prácticamente no se obtuvieron datos en este sentido. Por otra parte, como resulta común en los diseños cualitativos, las conclusiones no pueden ser consideradas representativas de todas las mujeres con discapacidad. De hecho, y a pesar de estar basadas en un conjunto amplio de experiencias, no se han incluido a mujeres con discapacidades sensoriales o intelectuales, que podrían ser objeto de futuros estudios. Tampoco se ha entrado a valorar otros factores que podrían 
incidir en la práctica y en el sentido de AFTO como la edad, el nivel de formación, ingresos o experiencias deportivas. Para ello sería recomendable profundizar en estudios de casos que ayudaran a identificar e interpretar cómo se enmarca la AFTO en la realidad situada de las PcD. Teniendo en cuenta estas limitaciones, nuestro trabajo se ha centrado en comprender mejor las experiencias de un colectivo todavía muy minoritario: el de mujeres con discapacidad practicantes de AFTO. Precisamente, con ello buscamos contribuir a que estas personas tengan más y mejores oportunidades de disfrutar de la AFTO en entornos adecuados.

\section{REFERENCIAS}

BRAUN, Virginia; CLARKE, Victoria. Using thematic analysis in psychology. Qualitative Research in Psychology, v. 3, n. 2, p. 77-101, 2006.

DEVÍS, José; PÉREZ-SAMANIEGO, Víctor. La ética en la promoción de la actividad física relacionada con la salud. In: DEVÍS, José. (Coord.). La educación física, el deporte y la salud en el siglo XXI. Alcoy: Marfil, 2001. p. 341-355.

DIBB, Bridget et al. Exploring positive adjustment in people with spinal cord injury. Journal of Health Psychology, v. 19, n. 8, p. 1043- 1054, ago. 2014.

INSTITUTO NACIONAL DE ESTADÍSTICA. Encuesta de Discapacidad, Autonomía personal y situaciones de Dependencia (EDAD). Madrid, 2008. Disponible en: <http://www.ine.es/prensa/ np524.pdf>. Acceso en: 20 ago. 2016.

EVANS, Adam; ALLEN-COLLINSON, Jacquelyne. From 'just a swimmer'to a 'swimming mother': women's embodied experiences of recreational aquatic activity with pre-school children. Leisure studies, v. 35, n. 2, p. 141-156, 2016.

HAGGER, Martin S.; ARMITAGE, Christopher J. The influence of perceived loci of control and causality in the theory of planned behavior in a leisure-time exercise context. Journal of Applied Biobehavioral Research, v. 9, n. 1, p. 45-64, ene. 2004.

HALLBERG, Inger et al. A striving for independence: a qualitative study of women living with vertebral fracture. BMC Nursing, v. 9, n. 7, abr. 2010. Disponible en: <http://www.biomedcentral. com/1472-6955/9/7>. Acceso en: 20 ago. 2016.

HAMMELL, Whalley K. Quality of life after spinal cord injury: a meta-synthesis of qualitative findings. Spinal Cord, v. 45, n. 2, p. 124-139, feb. 2007.

HICKS, Audrey L. et al. The effects of exercise training on physical capacity, strength, body composition and functional performance among adults with spinal cord injury: a systematic review. Spinal Cord, v. 49, n. 11, p. 1103-1127, nov. 2011.

LETTS, Lori et al. Preferred methods and messengers for delivering physical activity information to people with spinal cord injury: A focus group study. Rehabilitation Psychology, v. 56, n. 2, p. 128-137, may 2011.

MARTIN GINIS, Kathleen A. et al. Physical activity and subjective well-being among people with spinal cord injury: a meta-analysis. Spinal Cord, v. 48, n. 1, p. 65-72, jan.. 2010. 
MARTIN GINIS, Kathleen et al. A systematic review of review articles addressing factors related to physical activity participation among children and adults with physical disabilities. Health

Psychology Review, v.10, n.4, p.478-494, 2016.

MORRISEY, Barbara. Ethics and research among persons with disabilities in long-term care. Qualitative Health Research, v. 22, n. 9, p. 1284-1297, sept. 2012.

NEWITT, Rosemarie; BARNETT, Fiona; CROWE, Melissa. Understanding factors that influence participation in physical activity among people with a neuromusculoskeletal condition: a review of qualitative studies. Disability and Rehabilitation, v. 38, n. 1, p. 1-10, 2016.

PÉREZ-SAMANIEGO, Víctor; DEVÍS, José. Conceptuación y medida de las actitudes hacia la actividad física relacionada con la salud. Revista de Psicología del Deporte, v. 13, n. 2, p. 157173, oct. 2004.

PERRIER, Marie-Josée; SMITH, Brett; LATIMER-CHEUNG, Amy E. Stories that move? Peer athlete mentors' responses to mentee disability and sport narratives. Psychology of Sport and Exercise, v. 18, p. 60-67, may 2015.

RICHARDSON, Emma; SMITH, Brett; PAPATHOMAS, Anthony. Disability and the gym: experiences, barriers and facilitators of gym use for individuals with physical disabilities. Disability and Rehabilitation, Disponible en: < http://www.tandfonline.com/doi/full/10. 1080/09638288.2016.1213893>. Acceso en: 19 sept. 2016.

RIMMER, James H.; SCHILLER, William; CHEN, Ming-De. Effects of disability-associated low energy expenditure deconditioning syndrome. Exercise and Sport Sciences Reviews, v. 40, n. 1, p. 22-29, ene. 2012.

RYAN, Richard M.; DECl, Edward L. Self-determination theory and the facilitation of intrinsic motivation, social development, and well-being. American psychologist, v. 55, n. 1, p. 68-78, ene. 2000.

SCHWANDT, Thomas A. Qualitative inquiry: a dictionary of terms. Thousand Oaks: Sage, 1997.

SMITH, Brett; PERRIER, Marie-Josée. Disability, sport and impaired bodies. A critical approach. In: SCHINKE, Robert J.; MCGRANNON, Kerry R. (Eds.). The psychology of subculture in sport and physical activity: Critical perspectives. London: Routledge, 2014. p. 95-106.

SPARKES, Andrew; SMITH, Brett. Men, spinal cord injury, memories and the narrative performance of pain. Disability \& Society, v. 23, n. 7, p. 679-690, dic. 2008.

SPARKES, Andrew; SMITH, Brett. Qualitative research methods in Sport, Exercise and Health. Oxon: Routledge, 2014.

STATHI, Aphrodite ; FOX, Kenneth R. ; MCKENNA, James. Physical activity and dimensions of subjective well-being in older adults. Journal of Aging and Physical Activity, v. 10, n. 1, p. 76-92, ene. 2002.

STEPHENS, Catherine; NEIL, Rich; SMITH, Paul. The perceived benefits and barriers of sport in spinal cord injured individuals: A qualitative study. Disability and Rehabilitation, v. 34, n. 24, p. 2061-2070, abr. 2012. 
VAN DE VEN, Leontine et al. Strategies for autonomy used by people with cervical spinal cord injury: A qualitative study. Disability and Rehabilitation, v. 30, n. 4, p. 249-260, 2008.

WILLIAMS, Toni L.; SMITH, Brett; PAPATHOMAS, Anthony. The barriers, benefits and facilitators of leisure time physical activity among people with spinal cord injury: a meta-synthesis of qualitative findings. Health Psychology Review, v. 8, n. 4, p. 404-425, may 2014. 


\section{Agradecimientos:}

a las participantes, a Gabriel Brizuela y a los colaboradores de Tetrasport las facilidades que nos ofrecieron en todo momento para la realización de este trabajo. Asimismo, agradecemos a José Devís, Sofía Pereira, Joan Ubeda y al resto de miembros de la UTPAFIDE sus comentarios y apreciaciones sobre los borradores iniciales, así como a los dos revisores anónimos sus consideraciones sobre la versión final. 